\title{
Incorporation of mutations in five genes in the revised International Prognostic Scoring System can improve risk stratification in the patients with myelodysplastic syndrome
}

Hsin-An Hou', Cheng-Hong Tsai $\mathbb{1}^{1,2}$, Chien-Chin Lin ${ }^{1,3}$, Wen-Chien Chou ${ }^{1,3}$, Yuan-Yeh Kuo $\mathbb{B}^{4}$, Chieh-Yu Liu ${ }^{5}$, Mei-Hsuan Tseng ${ }^{1}$, Yen-Ling Peng ${ }^{1}$, Ming-Chih Liu', Chia-Wen Liư ${ }^{6}$, Xiu-Wen Liao ${ }^{2}$, Liang-In Lin? ${ }^{7}$, Ming Yao', Jih-Luh Tang ${ }^{1}$ and Hwei-Fang Tien ${ }^{1}$

\begin{abstract}
Gene mutations have not yet been included in the 2016 WHO classification and revised International Prognostic Scoring System (IPSS-R), which are now widely utilized to discriminate myelodysplastic syndrome (MDS) patients regarding risk of leukemia evolution and overall survival (OS). In this study, we aimed to investigate whether integration of gene mutations with other risk factors could further improve the stratification of MDS patients. Mutational analyses of 25 genes relevant to myeloid malignancies in 426 primary MDS patients showed that mutations of CBL, IDH2, ASXL1, DNMT3A, and TP53 were independently associated with shorter survival. Patients within each IPSSR or 2016 WHO classification-defined risk group could be stratified into two risk subgroups based on the mutational status of these five genes; patients with these poor-risk mutations had an OS shorter than others in the same risk group, but similar to those with the next higher risk category. A scoring system incorporating age, IPSS-R and five poor-risk mutations could divide the MDS patients into four risk groups $(P<0.001$ for both OS and leukemia-free survival). In conclusion, integration of gene mutations in current IPSS-R improves the prognostication of MDS patients and may help identify high-risk patients for more aggressive treatment in IPSS-R lower risk group.
\end{abstract}

\section{Introduction}

The myelodysplastic syndrome (MDS) is a heterogeneous group of clonal hematopoietic disorders characterized by various clinical presentations, risks for leukemia transformation and reduced survival ${ }^{1,2}$. This makes the accurate diagnosis, classification, and risk

Correspondence: H-A. Hou (hsinanhou@ntu.edu.tw) or H-F. Tien (hftien@ntu. edu.tw)

${ }^{1}$ Division of Hematology, Department of Internal Medicine, National Taiwan University Hospital, Taipei, Taiwan

${ }^{2}$ Tai-Cheng Stem Cell Therapy Center, National Taiwan University, Taipei, Taiwan

Full list of author information is available at the end of the article stratification of MDS patients clinically relevant ${ }^{3}$. A number of prognostic models, including International Prognostic Scoring System (IPSS), World Health Organization (WHO) based Prognostic Scoring System (WPSS), and the revised IPSS (IPSS-R), etc. have been developed to help risk-stratify these patients and facilitate clinical decision-making ${ }^{4-6}$.

Over the past decade, high throughput genetic techniques have uncovered novel genetic alterations and illuminated the genomic landscape of $\mathrm{MDS}^{7-10}$. Importantly, some of these mutations are associated with distinct clinical features and can predict survival ${ }^{11-14}$. 
Nevertheless, molecular data are not included in current prognostic systems. The 2001 WHO Classification included cytogenetics in MDS classification, and the updated 2016 WHO classification first includes the status of SF3B1 mutation in the classification of MDS with ring sideroblasts ${ }^{15,16}$.

Recently, Haferlach et al. ${ }^{12}$ and Nazha et al. ${ }^{17}$ demonstrated that integrating mutational profiling into the IPSS$\mathrm{R}$ could better discriminate MDS patients into various prognostic groups. However, the number of incorporated genes, patient population and treatment strategies were highly variable in these two studies. More investigations in the use of mutational data for MDS prognostication may give clearer view in this regard. Further, since the demographics and disease natures in MDS patients are different between Asian and Western countries ${ }^{18-20}$, it is unclear whether this new risk model combining both clinical parameters and genetic data are similarly useful for risk stratification of MDS patients in Asia. Besides, it remains to be answered whether the mutational status could further help improve the prognostic prediction in the 2016 WHO classification. In this study, we aimed to assess the prognostic impact of integration of gene mutations with conventional risk factors in a large cohort of patients with primary MDS in Taiwan. We found that patients within each IPSS-R or 2016 WHO classification-defined risk group could be stratified into two risk subgroups with different outcomes based on the mutational status of $C B L, I D H 2, A S X L 1$, DNMT3A, and TP53. Patients with these poor-risk mutations had an OS worse than others in the same risk group, but similar to those in the next higher risk group. Mutations screening in these five genes may help identify high-risk patients in IPSS-R lower risk group for more aggressive treatment.

\section{Patients and methods \\ Patient cohorts}

From 1984 to 2010, 426 adult patients with primary MDS who were diagnosed at the National Taiwan University Hospital (NTUH) and had complete clinical, mutational and cytogenetic data were included. Patients with secondary or therapy-related MDS were excluded to make the cohort more homogeneous. The diagnosis and classification of MDS were based on the French-American-British (FAB), 2008 and 2016 WHO criteria $^{15,16,21}$. Among them, the disease of 328 patients fulfilled the criteria of MDS according to the 2016 WHO classification. Most of the patients received conservative and supportive care. Twenty-two patients (5.2\%) were treated with acute myeloid leukemia (AML)-directed chemotherapy, $24(5.6 \%)$ patients received hypomethylating agents (HMA), and 46 (10.8\%) patients, allogeneic hematopoietic stem cell transplantation (HSCT). This study was approved by the institutional review board of the NTUH (20150709RINA) and written informed consent was obtained from all participants in accordance with the Declaration of Helsinki.

\section{Cytogenetics}

Bone marrow cells were collected directly or after 1-3 d of unstimulated culture as described previously ${ }^{22}$. Metaphase chromosomes were banded by trypsin-Giemsa technique and karyotyped according to the International System for Human Cytogenetic Nomenclature.

\section{Mutation analysis}

DNA was extracted from BM cells obtained at the time of diagnosis. Mutational analyses of 25 relevant genes involving in activated signaling pathways, such as FLT3$\mathrm{ITD}^{22}, N R A S^{22}, K R A S^{22}, J A K 2^{22}$, and $C B L ;{ }^{23}$ the transcription factors, such as RUNX1 ${ }^{24}$ and GATA2 ${ }^{25}$ splicing factors, including $S R S F 2^{26}, U 2 A F 1^{26}, S F 3 B 1^{26}$, and ZRSR2; ${ }^{27}$ epigenetic modifications, including $I D H 1^{28}$, $I D H 2^{28}, D N M T 3 A^{29}, T E T 2^{30}, M L L / \mathrm{PTD}^{31}, A S X L 1^{32}$ and $E Z H 2{ }^{33}$ cohesingenes $^{34}$, including RAD21, STAG1, STAG2, SMC1A, and SMC3, as well as SETBP $1^{35}$ and $T P 53^{36}$, were performed as previously described. Abnormal sequencing results were confirmed by at least two repeated analyses. To detect these mutations at diagnosis, we used DNA amplified in vitro from patients' bone marrow cells by Illustra ${ }^{\mathrm{TM}}$ GenomiPhi V2 DNA amplification kit as described by the manufacturer (GE Healthcare, Buckinghamshire, UK). All the mutations detected in such samples were verified in the original nonamplified samples.

\section{Statistical analysis}

The discrete variables of patients with and without poor-risk gene mutations were compared using the $\chi^{2}$ tests, but if the expected values of contingency tables were smaller than 5, Fisher exact test was used. Mann-Whitney test method was used to compare continuous variables and medians of distributions. Overall survival (OS) was measured from the date of first diagnosis to the date of last follow-up or death from any cause. Leukemia-free survival (LFS) was defined as the time from the date of MDS diagnosis to the date of AML evolution or death from any cause, whichever came first. Kaplan-Meier estimation was used to plot survival curves, and log-rank tests were used to calculate the difference of OS and LFS between groups. Multivariate Cox proportional hazard regression analysis was used to investigate independent prognostic factors for OS. The proportional hazards assumption (constant hazards assumption) was examined by using time-dependent covariate Cox regression before conducting multivariate Cox proportional hazard regression. The variables, including age, gender, IPSS-R, and 
Table 1 Mutation frequencies of $\mathbf{2 5}$ myeloid genes

\begin{tabular}{|c|c|c|c|}
\hline Variables & $\begin{array}{l}\text { No. } \\
\text { examined }\end{array}$ & Mutated no. & Percentage (\%) \\
\hline $\begin{array}{l}\text { RAS signaling } \\
\text { pathway }\end{array}$ & 426 & 37 & 8.7 \\
\hline NRAS & 426 & 19 & 4.5 \\
\hline KRAS & 426 & 6 & 1.4 \\
\hline$C B L$ & 426 & 13 & 3.1 \\
\hline Receptors/kinases & 426 & 9 & 2.1 \\
\hline FLT3/TD & 426 & 5 & 1.2 \\
\hline JAK2 & 426 & 4 & 0.9 \\
\hline Transcription & 426 & 61 & 14.3 \\
\hline RUNX1 & 426 & 52 & 12.2 \\
\hline GATA2 & 426 & 11 & 2.6 \\
\hline DNA methylation & 426 & 104 & 24.4 \\
\hline IDH1 & 426 & 4 & 0.9 \\
\hline $\mathrm{IDH} 2$ & 426 & 18 & 4.2 \\
\hline DNMT3A & 426 & 42 & 9.9 \\
\hline TET2 & 426 & 59 & 13.8 \\
\hline $\begin{array}{l}\text { Chromatin } \\
\text { modification }\end{array}$ & 426 & 103 & 24.2 \\
\hline ASXL1 & 426 & 96 & 22.5 \\
\hline$E Z H 2$ & 426 & 27 & 6.3 \\
\hline MLL/PTD & 426 & 5 & 1.2 \\
\hline RNA splicing & 426 & 166 & 39 \\
\hline U2AF1 & 426 & 31 & 7.3 \\
\hline SRSF2 & 426 & 58 & 13.6 \\
\hline SF3B1 & 426 & 47 & 11.0 \\
\hline ZRSR2 & 426 & 41 & 9.6 \\
\hline Cohesin & 426 & 31 & 7.3 \\
\hline RAD21 & 426 & 0 & 0 \\
\hline STAG1 & 426 & 2 & 0.5 \\
\hline STAG2 & 426 & 27 & 6.3 \\
\hline SMCIA & 426 & 2 & 0.5 \\
\hline SMC3 & 426 & 0 & 0 \\
\hline Others & 426 & 55 & 12.9 \\
\hline SETBP1 & 426 & 14 & 3.3 \\
\hline TP53 & 426 & 41 & 9.6 \\
\hline
\end{tabular}

CBL, RUNX1, IDH2, DNMT3A, TET2, ASXL1, EZH2, SRSF2, ZRSR2, TP53, and cohesin gene mutations were used as covariates. A $P$-value $<0.05$ was considered statistically significant. All statistical analyses were performed with SPSS 18 software (SPSS Inc., Chicago, IL, USA) and Statsdirect (Cheshire, England, UK).

\section{Results}

Supplementary Table 1 summarized the baseline characteristics of all enrolled patients. The median age at diagnosed was 67 years (range 18-98), with 58.9\% patients older than 65 years. According to the FAB classification, 155 patients (36.4\%) had refractory anemia (RA), 32 (7.5\%) had RA with ring sideroblasts (RARS), 141 (33.1\%) had RA with excess blasts (RAEB), 46 (10.8\%) had RAEB in transformation (RAEBT), and 52 (12.2\%), chronic myelomonocytic leukemia (CMML). In the 328 patients with MDS defined by the 2016 WHO classification, most had MDS with multilineage dysplasia (MDS-MLD, 25.3\%), followed by EB2 (22.3\%), EB1 (20.7\%) and MDS with single-lineage dysplasia (MDS-SLD, 20.1\%). By IPSS$\mathrm{R}, 27.7 \%$ of the entire cohort had lower risk (very low and low risk), 25.3\% had intermediate risk and 47\% had highrisk MDS (high and very high risk).

\section{Frequency and distribution of gene mutations}

In mutational analyses of 25 genes, 285 (66.9\%) of 426 patients harbored at least one molecular genetic alteration; whereas, cytogenetic studies identified karyotypic abnormalities in 37\%. Taken together, $77.4 \%$ patients had either gene mutation or cytogenetic change at diagnosis. One hundred and $82(42.7 \%)$ patients had two or more mutations. Unsurprisingly, the patients with advanced MDS, defined by the FAB, 2016 WHO classification or IPSS-R had more mutations compared with those with lower risk subtypes $(P<0.001$; Supplementary Figure 1$)$.

The frequent mutations with an incidence of $>10 \%$ were ASXL1 (22.5\%), TET2 (13.8\%), SRSF2 (13.6\%), RUNX1 (12.2\%), and SF3B1 mutations (11\%, Table 1 and Supplementary Figure 2). Less common mutations included DNMT3A (9.9\%), ZRSR2 (9.6\%), TP53 (9.6\%), U2AF1 (7.3\%), STAG2 (6.3\%), and EZH2 (6.3\%) mutations. Except SF3B1 and TET2 mutations, most of other mutations occurred more frequently in IPSS-R high- or very highrisk subgroup than in lower risk subgroups (Supplementary Figure 3). Together, the most common functional pathway involved in gene mutations in this MDS cohort was RNA splicing (39\%), followed by DNA methylation (24.4\%), chromatin modification (24.2\%), and transcription (14.3\%, Table 1 and Supplementary Figure 4).

Statistically significant positive or negative correlations were identified across 38 combinations of mutations, indicating the presence of co-operativeness and/or mutual exclusiveness of mutations in the pathogenesis of MDS (Fig. 1 and Supplementary Figure 5). In brief, mutations of genes involved in RNA splicing machinery or cohesin complex were mutually exclusive. On the other hand, ASXL1 mutation, the most prevalent mutation in our 


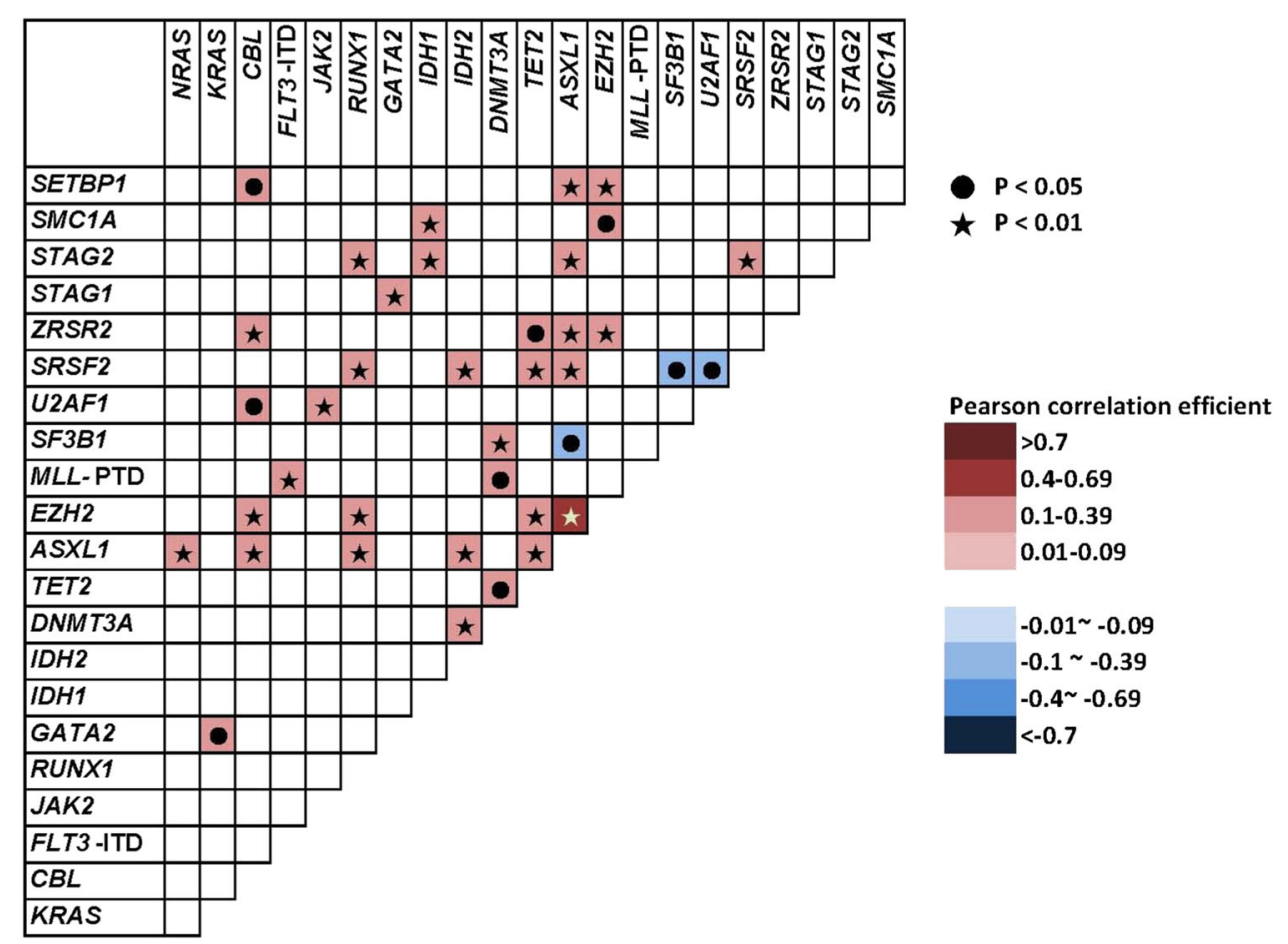

Fig. 1 Pairwise associations among gene mutations in 426 MDS patients

sample, frequently coexisted with other mutations; it significantly co-mutated with EZH2, STAG2, SRSF2, ZRSR2, TET2, IDH2, RUNX1, and SETBP1 mutations, but was exclusive with $S F 3 B 1$ mutation. TET2 mutation was positively associated with $E Z H 2, A S X L 1, S R S F 2$, and ZRSR2 mutations, RUNX1 mutation with EZH2, ASXL1, $S R S F 2$, and STAG2 mutations, and SRSF2 mutation with ASXL1, RUNX1, IDH2, TET2, and STAG2 mutations.

\section{Prognostic impact of mutations}

With a median follow-up of 43.2 months (range 0.1-250.7), the median OS for total cohort was 30.9 months. Because only 12 patients were of IPSS-R very low risk, we compiled the patients with IPSS-R very low and low risk together into survival analysis. Both OS and leukemia transformation rate were significantly different among the four subgroups of patients stratified by IPSS-R (both $P$-value <0.001; Supplementary Figure 6). We found that the clinical outcome got worse progressively as the patients had increasing number of somatic mutations (LFS and OS, $P<0.001$; Supplementary Figure 7).

In univariate analysis, older age, male gender, and IPSS$\mathrm{R}$ scores predicted prognosis in MDS patients. Besides, mutations in eleven genes, including $C B L, R U N X 1, I D H 2$, DNMT3A, TET2, ASXL1, EZH2, SRSF2, ZRSR2, Cohesin, and TP53 were significantly associated with reduced OS (Table 2). In the multivariate Cox proportional hazards regression analysis, older age and mutations in $C B L$, IDH2, DNMT3A, ASXL1, and TP53 were independent poor prognostic factors for OS, while lower IPSS-R scores had good prognostic impact (Table 3). Based on the above findings, we defined the five prognostic genes $(C B L, I D H 2$, DNMT3A, ASXL1, and TP53) as poor-risk mutations. Totally, 178 (41.8\%) patients harbored at least one poorrisk mutation. Compared with patients without poor-risk mutation, these patients were older, had higher WBC counts at diagnosis (Supplementary Table 2), higher risk of leukemia transformation (5-year leukemia transformation rate $61.7 \pm 7.1 \%$ vs. $22.1 \pm 4.1 \%, P<0.001$, Supplementary Figure 8) and shorter OS (median 15 months vs. 69.9 months, $P<0.001$ ). Interestingly, presence of poor-risk mutations could further stratify the IPSS-R low or very low-risk patients into two groups with distinct outcomes (median OS, 38.4 months for patients with poor-risk mutations vs. 102.8 months for those without, $P$ $=0.003$; Fig. 2a). The survival of the IPSS-R very low or low-risk patients who harbored poor-risk mutation was similar to the IPSS-R intermediate-risk patients. Similarly, there were distinct intra-group differences in OS based on the presence or absence of poor-risk mutations among patients with IPSS-R intermediate, high, and very highrisk MDS (Fig. 2b-d). The same was also true for 5-year leukemia transformation rate (Fig. $2 \mathrm{e}-\mathrm{h}$ ). In other words, patients in each IPSS-R risk subgroup who harbored these unfavorable mutations had OS worse than other patients 
Table 2 Univariate analysis for the overall survival and 5-year leukemia-free survival in MDS patients

\begin{tabular}{|c|c|c|c|c|c|c|}
\hline Variable & No. of patients & Median $\mathrm{OS} \pm \mathrm{SE}$, month & $P$-value & No. of patients & 5-year LFS (\%) & $P$-value \\
\hline Age & & & 0.001 & & & 0.556 \\
\hline$\geqq 65$ years & 221 & $26.3 \pm 3.6$ & & 199 & $68.1 \pm 6.1$ & \\
\hline$<65$ years & 205 & $39.7 \pm 16.5$ & & 181 & $60.4 \pm 5.2$ & \\
\hline Gender & & & 0.014 & & & 0.173 \\
\hline Male & 281 & $26.3 \pm 3.1$ & & 252 & $59.1 \pm 5.1$ & \\
\hline Female & 145 & $41.6 \pm 16.9$ & & 128 & $71.7 \pm 5.8$ & \\
\hline IPSS & & & $<0.001$ & & & $<0.001$ \\
\hline Low/Int1 & 249 & $69.9 \pm 12.4$ & & 249 & $73.5 \pm 4$ & \\
\hline Int2/high & 177 & $10.5 \pm 1.1$ & & 131 & $38.7 \pm 10.4$ & \\
\hline IPSS-R & & & $<0.001$ & & & $<0.001$ \\
\hline Very low/low & 118 & $83.6 \pm 15.2$ & & 118 & $88.2 \pm 4.1$ & \\
\hline Intermediate & 108 & $47.2 \pm 14.6$ & & 106 & $60.8 \pm 7.1$ & \\
\hline High & 106 & $17.7 \pm 1.2$ & & 86 & $52.6 \pm 7.8$ & \\
\hline Very high & 94 & $7.8 \pm 0.6$ & & 70 & $29.6 \pm 14.1$ & \\
\hline \multicolumn{7}{|l|}{ Mutations } \\
\hline NRAS & & & 0.114 & & & 0.001 \\
\hline Mutated & 19 & $31.9 \pm 2.6$ & & 11 & $31.8 \pm 17.5$ & \\
\hline Wild type & 407 & $15.9 \pm 3.6$ & & 369 & $66.3 \pm 4.1$ & \\
\hline KRAS & & & 0.524 & & & 0.302 \\
\hline Mutated & 6 & $17.7 \pm 4.6$ & & 5 & $53.3 \pm 24.8$ & \\
\hline Wild type & 420 & $31.1 \pm 2.8$ & & 375 & $65.5 \pm 3.7$ & \\
\hline$C B L$ & & & 0.001 & & & 0.009 \\
\hline Mutated & 13 & $11.8 \pm 2.1$ & & 12 & $38.6 \pm 17.3$ & \\
\hline Wild type & 413 & $32.5 \pm 2.7$ & & 368 & $66.1 \pm 3.8$ & \\
\hline FLT3/ITD & & & 0.289 & & & 0.003 \\
\hline Mutated & 5 & $16.9 \pm 5.7$ & & 4 & $25.0 \pm 21.7$ & \\
\hline Wild type & 421 & $31.3 \pm 2.6$ & & 376 & $65.6 \pm 3.8$ & \\
\hline$J A K 2$ & & & 0.989 & & & 0.396 \\
\hline Mutated & 4 & $19 \pm 7.0$ & & 3 & 100 & \\
\hline Wild type & 422 & $30.9 \pm 2.8$ & & 373 & $64.9 \pm 3.7$ & \\
\hline RUNX1 & & & 0.008 & & & $<0.001$ \\
\hline Mutated & 52 & $18.6 \pm 4.0$ & & & $34.6 \pm 10.4$ & \\
\hline Wild type & 374 & $32.5 \pm 2.4$ & & & $68.8 \pm 3.9$ & \\
\hline GATA2 & & & 0.150 & & & 0.903 \\
\hline Mutated & 11 & NA & & 9 & $65.6 \pm 20.9$ & \\
\hline Wild type & 415 & $30.9 \pm 2.8$ & & 371 & $65.1 \pm 3.8$ & \\
\hline $\mathrm{IDH} 1$ & & & 0.285 & & & 0.402 \\
\hline Mutated & 4 & $15 \pm 3.5$ & & 2 & 0 & \\
\hline Wild type & 422 & $31.9 \pm 2.5$ & & 378 & $65.4 \pm 3.7$ & \\
\hline
\end{tabular}


Table 2 continued

\begin{tabular}{|c|c|c|c|c|c|c|}
\hline Variable & No. of patients & Median $\mathrm{OS} \pm \mathrm{SE}$, month & $P$-value & No. of patients & 5-year LFS (\%) & $P$-value \\
\hline IDH2 & & & 0.02 & & & 0.081 \\
\hline Mutated & 18 & $18.5 \pm 6.6$ & & 13 & $56 \pm 17.1$ & \\
\hline Wild type & 408 & $32.6 \pm 2.5$ & & 367 & $65.9 \pm 3.8$ & \\
\hline DNMT3A & & & 0.035 & & & 0.137 \\
\hline Mutated & 42 & $16.9 \pm 3.9$ & & 37 & $33 \pm 23.8$ & \\
\hline Wild type & 384 & $32.7 \pm 2.8$ & & 343 & $65.3 \pm 4.0$ & \\
\hline TET2 & & & 0.022 & & & 0.305 \\
\hline Mutated & 59 & $20.1 \pm 3.2$ & & 57 & $60.2 \pm 10.1$ & \\
\hline Wild type & 367 & $31.9 \pm 2.9$ & & 323 & $65.8 \pm 4.0$ & \\
\hline ASXL1 & & & $<0.001$ & & & $<0.001$ \\
\hline Mutated & 96 & $18.7 \pm 2.6$ & & 84 & $30.8 \pm 9.7$ & \\
\hline Wild type & 330 & $36.3 \pm 8.1$ & & 296 & $73.2 \pm 3.8$ & \\
\hline EZH2 & & & 0.044 & & & $<0.001$ \\
\hline Mutated & 27 & $17 \pm 2.2$ & & 25 & $36.4 \pm 14.1$ & \\
\hline Wild type & 399 & $32.5 \pm 2.7$ & & 355 & $67.1 \pm 3.8$ & \\
\hline MLL/PTD & & & 0.149 & & & 0.018 \\
\hline Mutated & 5 & $16.9 \pm 0.6$ & & 3 & 0 & \\
\hline Wild type & 421 & $31.1 \pm 2.8$ & & 377 & $66.7 \pm 3.8$ & \\
\hline U2AF1 & & & 0.316 & & & 0.1 \\
\hline Mutated & 31 & $31.3 \pm 4.9$ & & 27 & $13.9 \pm 12.4$ & \\
\hline Wild type & 395 & $30.9 \pm 3.1$ & & 353 & $69.5 \pm 3.4$ & \\
\hline SRSF2 & & & 0.001 & & & 0.014 \\
\hline Mutated & 58 & $18.5 \pm 3.7$ & & 52 & $24.6 \pm 18.5$ & \\
\hline Wild type & 368 & $33.8 \pm 3.3$ & & 328 & $68.3 \pm 3.8$ & \\
\hline SF3B1 & & & 0.482 & & & 0.645 \\
\hline Mutated & 47 & $39.7 \pm 11.3$ & & 43 & $70.6 \pm 9.8$ & \\
\hline Wild type & 379 & $29.3 \pm 3.1$ & & 337 & $64.4 \pm 4$ & \\
\hline ZRSR2 & & & 0.008 & & & 0.525 \\
\hline Mutated & 41 & $14 \pm 2.1$ & & 37 & $63.7 \pm 11$ & \\
\hline Wild type & 385 & $32.7 \pm 2.9$ & & 343 & $65.2 \pm 3.9$ & \\
\hline SETBP1 & & & 0.988 & & & 0.163 \\
\hline Mutated & 14 & $36.4 \pm 16.8$ & & 13 & $45.5 \pm 16.6$ & \\
\hline Wild type & 412 & $31.1 \pm 2.7$ & & 367 & $65.7 \pm 3.8$ & \\
\hline TP53 & & & $<0.001$ & & & $<0.001$ \\
\hline Mutated & 41 & $6.6 \pm 1.2$ & & 29 & $41.3 \pm 11.9$ & \\
\hline Wild type & 285 & $36.1 \pm 3.0$ & & 351 & $67.2 \pm 3.8$ & \\
\hline Cohesin $^{a}$ & & & 0.005 & & & $<0.001$ \\
\hline Mutated & 31 & $22.5 \pm 4.3$ & & 23 & $21.5 \pm 12.2$ & \\
\hline Wild type & 395 & $33.8 \pm 3.4$ & & 357 & $68.1 \pm 3.8$ & \\
\hline
\end{tabular}

OS overall survival, $C$ confidence interval, IPSS international prognostic scoring system, IPSS-R revised IPSS, LFS leukemia-free survival ${ }^{a}$ Cohesin genes, including RAD21, STAG1, STAG2, SMC1A, and SMC3 
Table 3 Multivariate analysis (Cox regression) for the overall survival and leukemia-free survival in MDS patients

\begin{tabular}{|c|c|c|c|c|c|c|c|c|}
\hline \multirow[t]{3}{*}{ Variables } & \multicolumn{4}{|c|}{ Overall survival } & \multicolumn{4}{|c|}{ Leukemia-free survival } \\
\hline & \multirow[b]{2}{*}{ RR } & \multicolumn{2}{|l|}{$95 \% \mathrm{Cl}$} & \multirow[b]{2}{*}{$P$-value } & \multirow[b]{2}{*}{ RR } & \multicolumn{2}{|l|}{$95 \% \mathrm{Cl}$} & \multirow[b]{2}{*}{$P$-value } \\
\hline & & Lower & Upper & & & Lower & Upper & \\
\hline Age & 1.024 & 1.014 & 1.034 & $<0.001^{*}$ & 0.997 & 0.983 & 1.011 & 0.713 \\
\hline Gender (male vs. female) & 1.248 & 0.922 & 1.687 & 0.151 & 1.251 & 0.753 & 2.079 & 0.387 \\
\hline IPSS-R scores ${ }^{a}$ & 0.306 & 0.208 & 0.450 & $<0.001^{*}$ & 0.211 & 0.102 & 0.437 & $<0.001^{*}$ \\
\hline CBL mutation & 2.292 & 1.164 & 4.513 & $0.016^{*}$ & 2.561 & 0.943 & 6.955 & 0.065 \\
\hline RUNX1 mutation & 1.021 & 0.679 & 1.536 & 0.920 & 1.381 & 0.729 & 2.617 & 0.322 \\
\hline IDH2 mutation & 1.957 & 1.045 & 3.665 & $0.036^{*}$ & 1.798 & 0.577 & 5.602 & 0.311 \\
\hline DNMT3A mutation & 1.571 & 1.028 & 2.401 & $0.037^{*}$ & 1.789 & 0.875 & 3.658 & 0.111 \\
\hline TET2 mutation & 1.233 & 0.829 & 1.835 & 0.301 & 1.270 & 0.642 & 2.513 & 0.493 \\
\hline ASXL1 mutation & 1.557 & 1.040 & 2.329 & $0.031^{*}$ & 2.009 & 1.066 & 3.788 & $0.031^{*}$ \\
\hline EZH2 mutation & 1.292 & 0.724 & 2.304 & 0.385 & 1.413 & 0.587 & 3.402 & 0.440 \\
\hline SRSF2 mutation & 1.084 & 0.684 & 1.719 & 0.731 & 1.168 & 0.530 & 2.576 & 0.700 \\
\hline ZRSR2 mutation & 1.212 & 0.782 & 1.879 & 0.389 & 1.035 & 0.477 & 2.249 & 0.930 \\
\hline Cohesin mutation ${ }^{\mathrm{b}}$ & 1.232 & 0.764 & 1.986 & 0.392 & 2.620 & 1.281 & 5.359 & $0.008^{*}$ \\
\hline TP53 mutation & 9.524 & 6.067 & 14.950 & $<0.001^{*}$ & 14.669 & 6.664 & 32.288 & $<0.001^{*}$ \\
\hline
\end{tabular}

$R R$ relative risk, $C l$ confidence interval, IPSS- $R$ revised international prognostic scoring system

*P-value $<0.05$ was considered significant

aIPSS-R scores: lower IPSS-R scores (very low- and low risk) vs. others

${ }^{\mathrm{b}}$ Cohesin genes, including RAD21, STAG1, STAG2, SMC1A, and SMC3A

of the same risk but similar to those in the next higher risk subgroup. Although the poor-risk mutations had significant impact on clinical outcomes in all IPSS-R risk groups, it was much obvious in lower risk population. Intriguingly, the molecular data could further reclassify $22.9 \%(27 / 118)$ of IPSS-R very low/low risk patients to intermediate risk subgroup, 31.5\% (34/108) of IPSS-R intermediate to high-risk subgroup, and 53.8\% (57/106) of IPSS-R high to very high-risk subgroup.

Next, we investigated the impact of molecular data on clinical outcomes in MDS patients according to the 2016 WHO classification. We found the distinct survival difference of MDS patients stratified by the 2016 WHO classification (Supplementary Figure 9). The median OS was 170.2, 57.6, 21.3, and 10.1 months for MDS-SLD, MDS-MLD, EB1, and EB2, respectively $(P<0.001)$. The 5year leukemia transformation rate was $17.2 \%, 24 \%, 45.1 \%$, and $78.6 \%$, respectively, for these four subgroups. Intriguingly, in the cohort of patients with MDS-SLD or MDSMLD, poor-risk mutations could further stratify these patients into two risk groups; the patients with unfavorable mutations had higher probability of leukemia transformation and poorer OS than those without in the same subgroup, but comparable to those with MDS-EB1 (Fig. 3a, b). Similarly, in the cohort of patients with MDS-EB1, those with poor-risk mutations had worse clinical outcomes regarding both leukemia transformation and OS, but comparable to the next higher risk subgroup with MDS-EB2 (Fig. 3c, d). The same was also true for MDSEB2 patients (Fig. 3e, f). However, presence of poor-risk mutations had no statistically significant impact on prognosis in the 34 patients with MDS-RS-SLD or MDSRS-MLD, possibly due to limited number of patients in this group.

To better stratify the MDS patients into different risk groups, an integrated scoring system incorporating seven prognostic markers, including age, IPSS-R, and mutations of $C B L, I D H 2, D N M T 3 A, A S X L 1$, and TP53, into survival analysis was formulated. A new risk model was developed incorporating the weighted coefficients of these factors: age $\times 0.025$ - IPSS-R lower risk group $\times 1.184+C B L \times$ $0.829+I D H 2 \times 0.829+D N M T 3 A \times 0.452+A S X L 1 \times$ $0.442+T P 53 \times 2.254$. Four risk groups were proposed: low (score $<-0.5 ; n=84$ ), intermediate (score $-0.5 \sim 0.5$; $n=158$ ), high (score $0.51 \sim 1.5 ; n=129$ ) and very high (score $>1.5 ; n=55$ ). The median OS was 250.7, 38.4, 17, and 8.9 months for low, intermediate, high, and very high subgroups, respectively. This clinically relevant integrated scoring system divided the MDS patients into four groups with different clinical outcomes $(P<0.001$ for both OS 
A

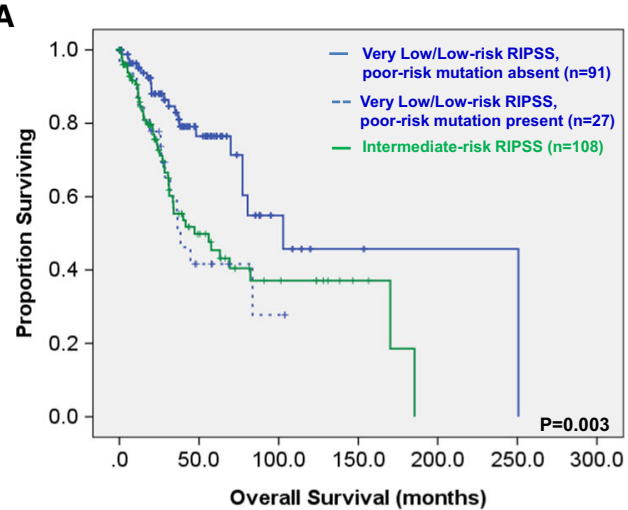

C

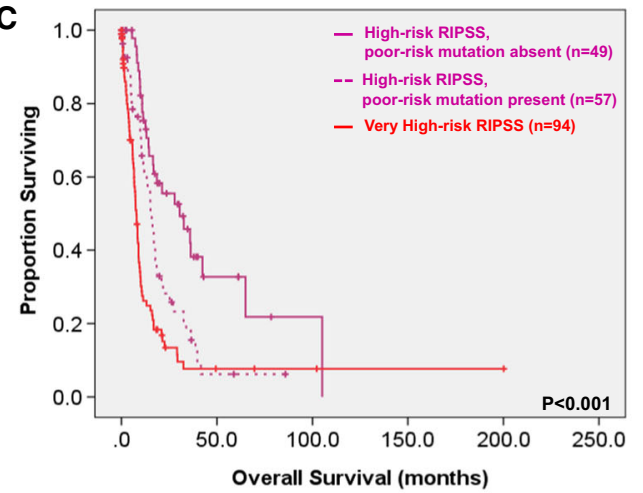

E

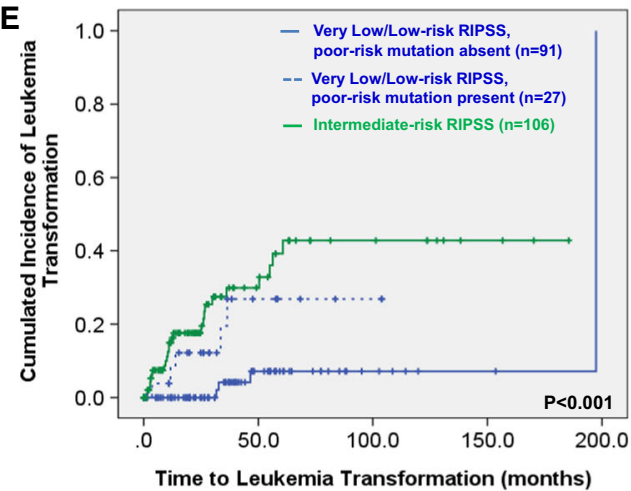

G

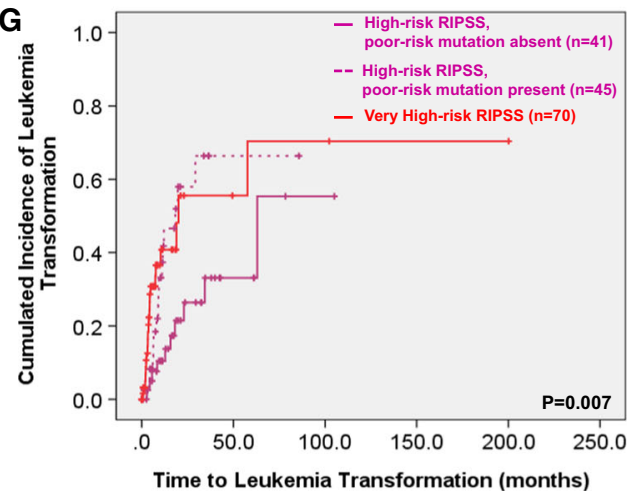

B

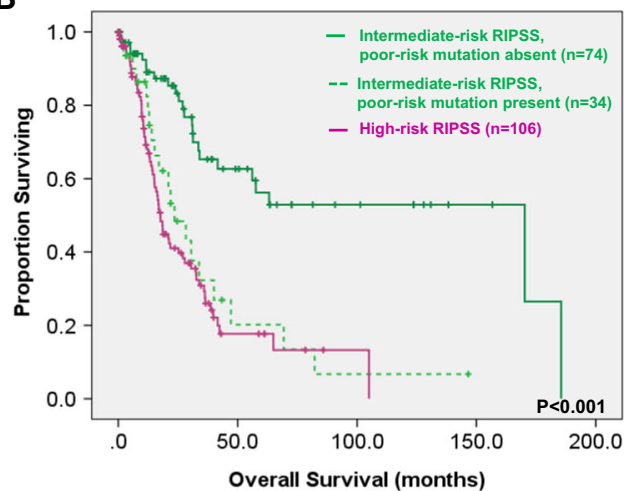

D

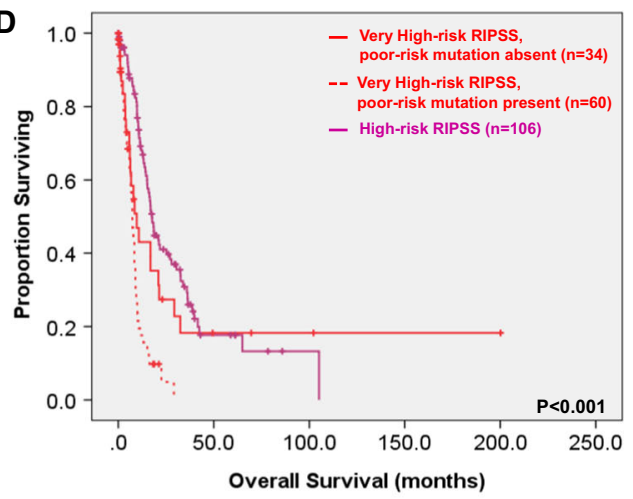

$F$
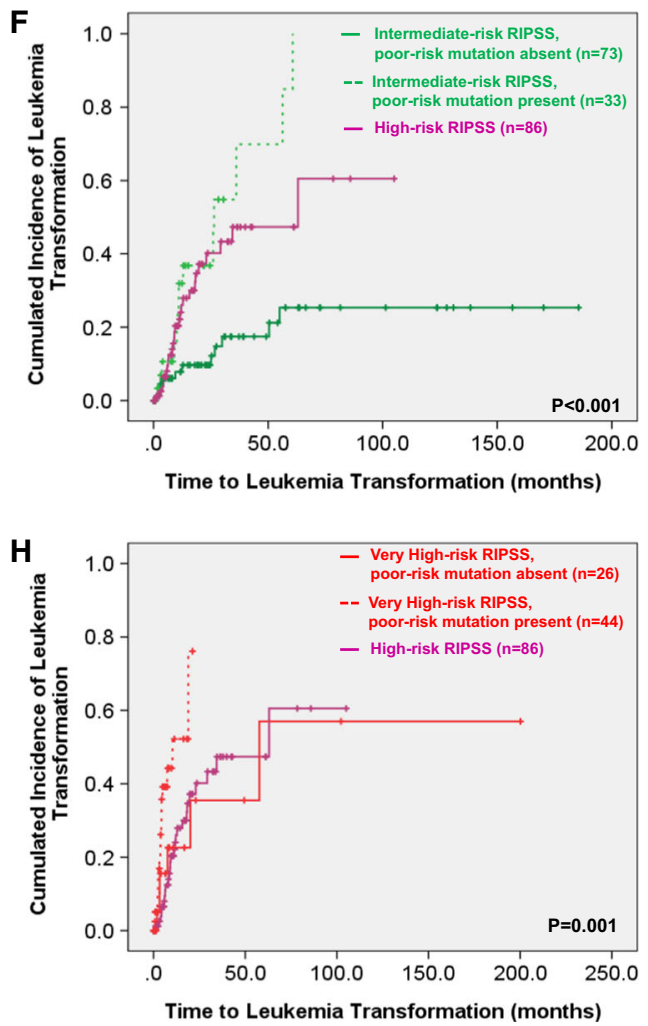

Fig. 2 Overall survival and leukemia transformation rate according to the revised International Prognostic Scoring System (IPSS-R) risk categories and mutational status. a-d Overall survival according to the presence or absence of poor-risk mutations (CBL, IDH2, ASXL1, DNMT3A, and TP53 mutations) and IPSS-R. e-h Leukemia transformation rate according to the presence or absence of poor-risk mutations (CBL, IDH2, ASXL1, DNMT3A, and TP53 mutations) and IPSS-R 

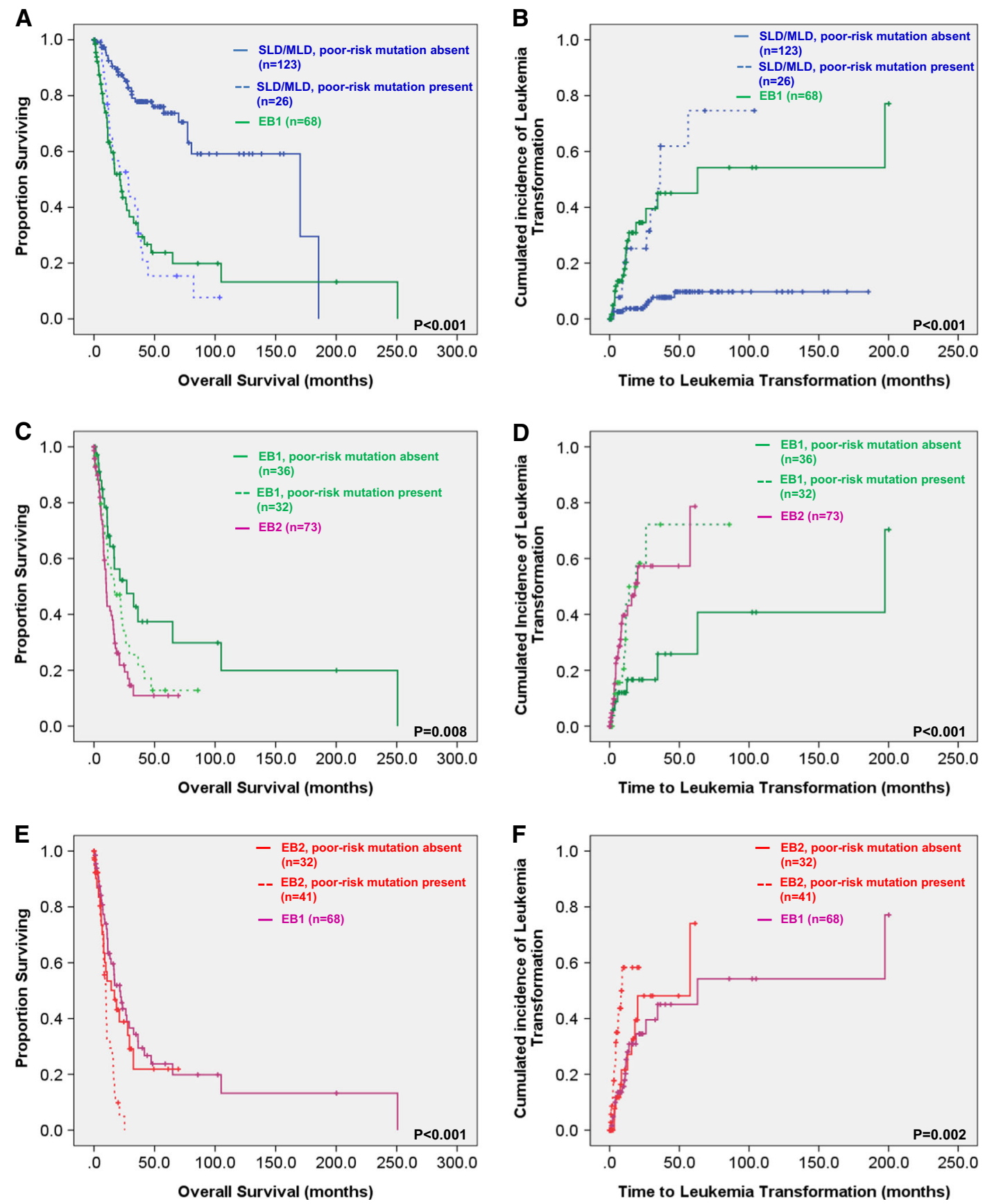

Fig. 3 Overall survival and leukemia transformation rate according to 2016 WHO classification risk categories and mutational status. a, c, e Overall survival according to the presence or absence of poor-risk mutations (CBL, IDH2, ASXL1, DNMT3A, and TP53 mutations) and 2016 WHO classification. b, d, $\mathbf{f}$ Leukemia transformation rate according to the presence or absence of poor-risk mutations (CBL, IDH2, ASXL1, DNMT3A, and TP53 mutations) and 2016 WHO classification

and LFS; Fig. 4). The same was also true for the 326 patients based on the 2016 WHO classification $(P<0.001$ for both OS and LFS; Fig. 5).

Finally, since some patients in our cohort received allogeneic HSCT, we evaluated the impact of the treatment on OS in individuals with poor-risk mutations. The median OS was significantly longer in the patients receiving allogeneic HSCT than those without in this high-risk group (91.4 months vs. 13.7 months, $P=0.01$; Supplementary Figure 10). In the cohort of patients with IPSS-R lower risk but unfavorable molecular genotype, the OS was similar to those without poor-risk mutation if 


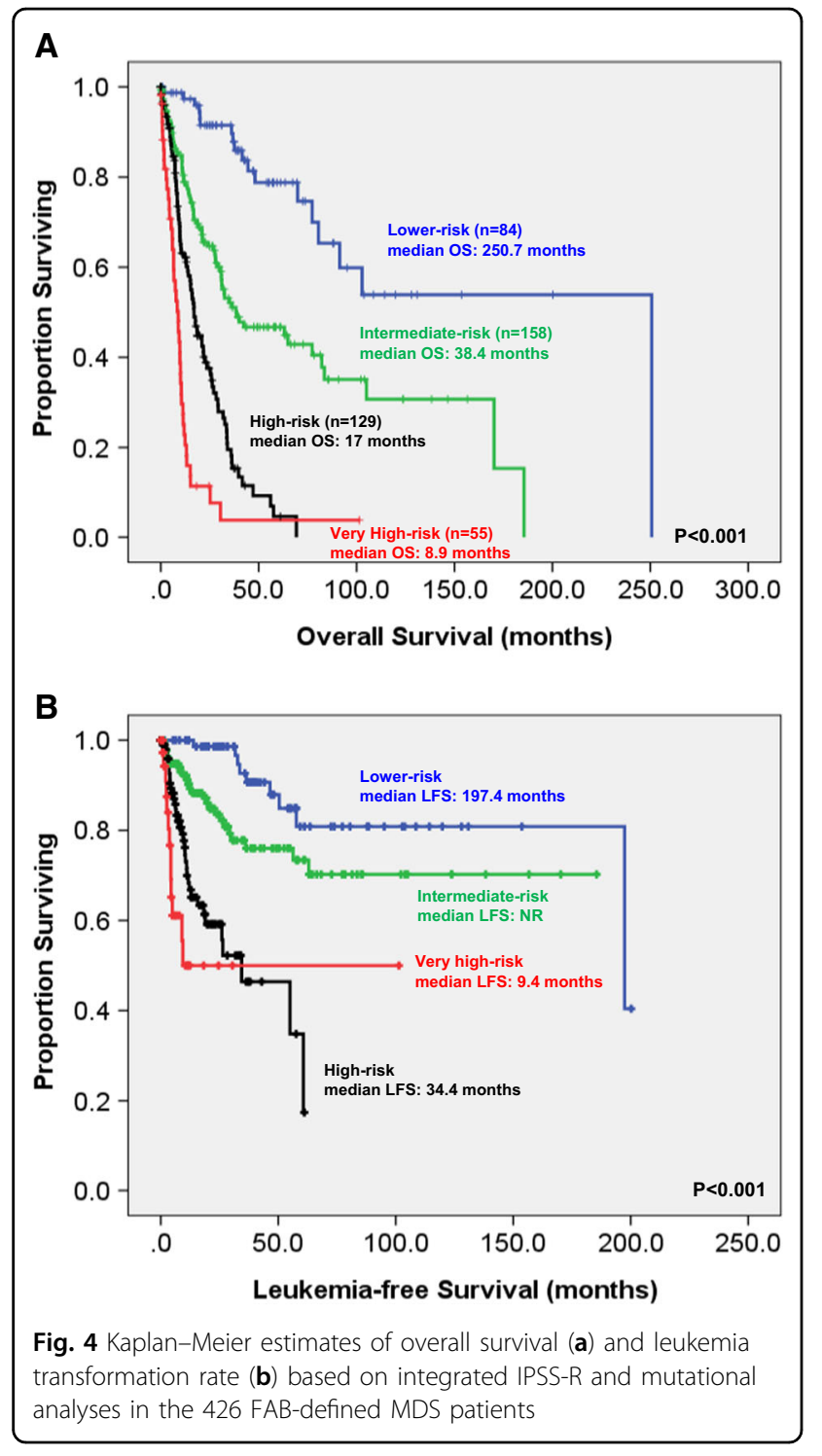

they received allogeneic HSCT $(P=0.452)$. Similar finding was also found in IPSS-R higher risk cohort $(P=$ 0.4902).

\section{Discussion}

To our knowledge, there have been few studies integrating molecular data into IPSS-R in MDS patients $^{12,17,37}$. Herein, we comprehensively analysed clinical parameters and 25 genetic alterations in a large series of MDS patients and found incorporation of mutational profiles into current IPSS-R and 2016 WHO classification may improve the prognostic stratification of MDS patients.

Because, the median survival and risk of leukemia transformation vary widely in the MDS patients, even in the same IPSS or IPSS-R group, it is prudent to identify novel prognostic markers to make the prediction more
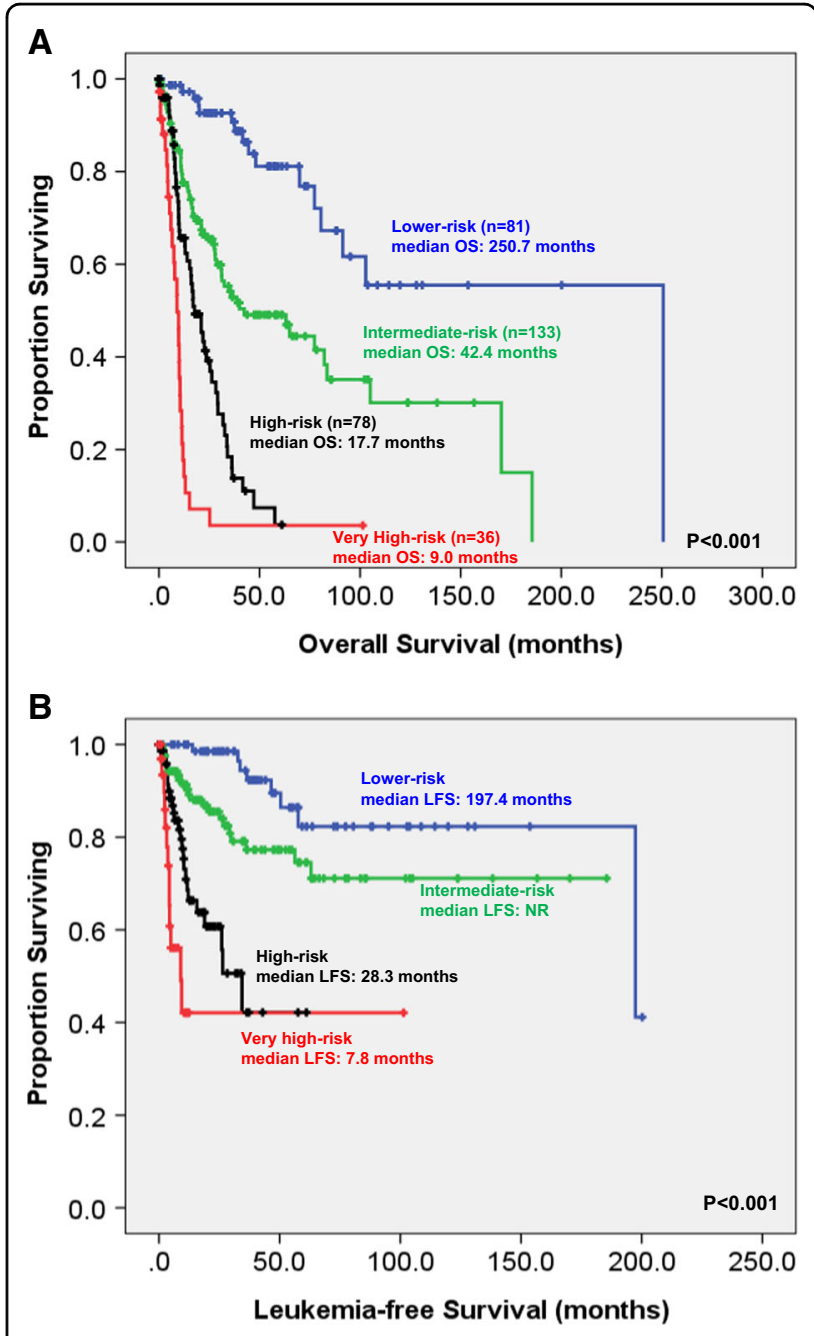

Fig. 5 Kaplan-Meier estimates of overall survival (a) and leukemia transformation rate (b) based on integrated IPSS-R and mutational analyses in the $328 \mathrm{WHO}$-defined MDS patients

accurate. Bejar et al. ${ }^{8}$ first proposed a prognostic model using both IPSS and the mutation status of RUNX1, ETV6, EZH2, ASXL1, and TP53 to provide better prognostic prediction. Similarly, combination of $E Z H 2$ mutation and MD Anderson lower risk prognostic scoring system could identify $29 \%$ of lower risk MDS patients with a worse-than-expected prognosis ${ }^{38}$. However, a growing list of molecular alterations, such as RNA splicing genes and cohesin complex mutations, etc., were detected in MDS recently after these prior reports, and it is getting clear that some of these newly identified gene mutations have distinct phenotype and prognostic relevance ${ }^{39}$. In a mutational study of 111 genes in 738 patients with MDS or closely related neoplasm, Papaemmanuil et al. ${ }^{11}$ showed the total number of oncogenic mutations and cytogenetic lesions were negatively associated with LFS. Among them, eight mutations, including CUX1, BCOR, 
RUNX1, IDH2, ASXL1, U2AF1, SRSF2, and TP53 mutations predicted shorter LFS while SF3B1 mutation was associated with better LFS. Further, the numbers of driver mutations could further provide independent prognostic impact on LFS in patients with IPSS low and Int-1 risk.

Compared with IPSS, the IPSS-R incorporated more sophisticated parameters, including blasts percentages, cytogenetic classification and the depth of cytopenias to improve prognosis assessment for MDS patients ${ }^{6}$. IPSS-R has been the standard tool to risk stratify MDS patients. Haferlach et al. ${ }^{12}$ utilized a combination of conventional factors (age, gender, and IPSS-R) and mutations in 14 genes (CBL, NARS, KRAS, ETV6, NPM1, LAMB4, NF1, PRPF8, RUNX1, TET2, ASXL1, EZH2, STAG2, and TP53) as a novel prognostic model. It separated the patients into four risk groups ${ }^{12}$. In a study of Nazha et al., ${ }^{17}$ incorporation of three mutations (EZH2, SF3B1, and TP53) into IPSS- $\mathrm{R}$ can improve the predictive power in 508 patients with primary and secondary MDS. Recently, Tefferi et al. ${ }^{37}$ showed that the survival impact of three adverse mutations, including ASXL1, TP53, and SRSF2 mutations was most evident in IPSS-R very low- and lowrisk patients, implying targeted sequencing may assist in decision-making in management of this group. However, the disease subtypes, adverse mutations identified and therapy choices were quite variable in these studies. More investigations are required to get clearer view. Further, the implication of mutations on clinical outcomes in Asian MDS patients may be different from that in western patients since disease natures of MDS and racial background are different between these two groups ${ }^{18-20,32}$. The current study was aimed to assess the additive prognostic impact of molecular profiling on IPSS-R and 2016 WHO classification in a large cohort of MDS patients in Asia. Further, an integrated risk-stratification model incorporating conventional risk factors and gene mutations was established to better risk-stratify MDS patients. We found LFS and OS were negatively associated with the number of mutations, compatible with the concept that clonal evolution with acquisition of genetic changes is associated with disease progression and poor survival $^{40}$.

The association between specific cytogenetic change and particular mutation pattern, such as TP53 with monosomal karyotype, RUNX1 with trisomy 21 and SF3B1 with inv $^{3}(\mathrm{q} 21 \mathrm{q} 26.2)$ and $\operatorname{del}(11 \mathrm{q})$ in MDS was recently reported, suggesting that mutation-induced genomic instability may result in specific cytogenetic abnormality $^{41}$. Besides, the frequent co-existence of some mutations further provides the insights into molecular circuitry and mutual exclusiveness usually implies the functional redundancy in the development of $\operatorname{MDS}^{11,39}$. Most importantly, we showed that within each IPSS-R or 2016 WHO classification-defined risk group, two subgroups with different outcomes could be separated through mutational screening of five genes, including CBL, IDH2, ASXL1, DNMT3A, and TP53. Patients with these poor-risk mutations had an OS worse than others in the same IPSS-R or 2016 WHO subgroup, but similar to those in the next higher risk subgroup. These findings explain the clinical heterogeneity in the same IPSS-R risk groups. A substantial portion of patients in each IPSS- $R$ risk group could be adjusted to different prognostic groups based on the integrated prognostic system; $22.9 \%$ of the IPSS-R very low- and low-risk patients could be redistributed into intermediate risk group, 31.5\% of IPSS$\mathrm{R}$ intermediate risk patients to high-risk group, and 53.8\% of IPSS-R high-risk to very high-risk group. The incorporation of the status of these poor-risk mutations into the survival analysis would be especially helpful to identify patients with poorer prognosis for more aggressive treatment in the rather heterogeneous group of patients with lower risk MDS defined by conventional scoring systems.

The prognostic relevance of individual mutations in these five genes was largely comparable to those reported previously ${ }^{8,12,42-44}$. In contrast to the report of Haferlach et al. ${ }^{12}$, we could not find the independently prognostic relevance of RUNX1 mutation, TET2 mutation and STAG2 mutation in our cohort although these three mutations were associated with poorer OS in univariate analysis, most likely owing to strong associations between these three mutations and older age and advanced IPSS$\mathrm{R}^{45,46}$. On the other hand, IDH2 and DNMT3A mutations were poor-risk genotypes in our cohort, but not in that of Haferlach et $\mathrm{al}^{12}$. Although, SF3B1 mutation predicted better survival in the studies of Haferlach et al. ${ }^{12}$ and Nazha et al. ${ }^{17}$, SF3B1 mutation was not a prognostic factor in our cohort. The possible explanation was SF3B1 mutation was closely associated with older age and $D N M T 3 A$ mutation, two independently poor prognostic markers. The different results obtained from these studies were probably due to differences in the patients recruited and in the treatment strategies. In the study of Haferlach et al. ${ }^{12}$, patients were relatively older (median 72.8 years) and $77.1 \%$ received supportive care; while in the study of Nazha et al. ${ }^{17}$, both primary and secondary MDS patients were recruited and only 20\% received supportive care. Compared with these two reports, fewer cases were in IPSS-R very low- and low-risk groups in this study (27.7\% vs. $54 \%$ and $54.5 \%$ ). In contrast, about one half of patients had high or very high-risk MDS in our cohort.

The ultimate goal of management of MDS patients is to explore personalized therapy, thereby improve the treatment outcome while reduce the probability of leukemia transformation and treatment-related toxicity. Though most patients in this study only received palliative care, some did receive allogeneic HSCT, which gave us a 
chance to investigate the impact of genetic data on prognosis in patients undergoing this disease-modifying therapy. We previously showed that allogeneic HSCT could provide survival benefits for patients with IPSS-R high and very high risk ${ }^{20}$. However, the treatment choice for IPSS-R lower risk patients with documented poor-risk mutations, such as $C B L, I D H 2, A S X L 1, D N M T 3 A$, and TP53 mutations is currently unclear. We showed that patients with unfavorable molecular genotypes had a better OS if they received allogeneic HSCT than those who did not. We also found allogeneic HSCT could significantly improve the survival of patients with IPSS-R lower risk, but with poor-risk mutations. It implied that HSCT might ameliorate the poor survival impact of the adverse-risk genotypes. Further prospective studies with more patients recruited are needed to verify this point.

The limitation in our study is that we also included patients with RAEBT and CMML defined by FAB classification. However, we distinctly showed that mutational characterization and integrated scoring system could well risk-stratify not only FAB-defined MDS patients, but also those defined by WHO classification. Second, with the limited number of patients receiving HMA treatment in our cohort, we could not determine whether HMA could provide survival benefits for these poor-risk patients.

In summary, early assessment of IPSS- $\mathrm{R}$ and mutational profiling of five relevant genes, including $C B L, I D H 2$, $A S X L 1, D N M T 3 A$, and TP53, may improve the prognostic stratification of MDS patients. Presence of these poor-risk mutations can also risk-stratify the patients independently of 2016 WHO classification. The clinically relevant integrated prognostic system further refines the prognostic prediction models and may guide the therapeutic decision. Supplementary information is available at Blood Cancer Journal's website.

\section{Acknowledgements}

We would like to acknowledge the service provided by the DNA Sequencing Core of the First Core Laboratory, National Taiwan University College of Medicine. This work was partially sponsored by grants MOST 103-2628-B-002003-MY3, 103-2628-B-002-003-MY3, 104-2314-B-002-128-MY4, 106-2314-B-002226-MY3, and 106-2314-B-002-231-MY3 from the Ministry of Science and Technology (Taiwan), National Taiwan University Hospital-National Taiwan University joint research grant (UN103-051), and MOHW 105-TDU-B-211134004 from the Ministry of Health and Welfare (Taiwan), NTUH 102P06, from the Department of Medical Research, National Taiwan University Hospital, and Taiwan Health Foundation.

\section{Author contributions:}

H.-A.H. was responsible for study design, plan and coordination, literature collection, data management and interpretation, statistical analysis and manuscript writing; C.-Y.L. was responsible for statistical analysis and interpretation of the statistical findings; Y.-Y.K., L.I.L. was responsible for mutation analysis and interpretation; H.-A.H., C.-H.T., C.-C.L., X.-W.L., W.-C.C., M.Y., J.-L.T., and H.F.T. contributed patient samples and clinical data; M.-H.T., Y.-L.P., C.-W.L., and M.-C.L. performed the gene mutation and chromosomal studies and H.F.T. planned, designed, coordinated the study over the entire period and wrote the manuscript.

\begin{abstract}
Author details
'Division of Hematology, Department of Internal Medicine, National Taiwan University Hospital, Taipei, Taiwan. ${ }^{2}$ Tai-Cheng Stem Cell Therapy Center, National Taiwan University, Taipei, Taiwan. ${ }^{3}$ Departments of Laboratory Medicine, National Taiwan University Hospital, Taipei, Taiwan. ${ }^{4}$ Graduate Institute of Oncology, College of Medicine, National Taiwan University, Taipei, Taiwan. ${ }^{5}$ Biostatistics Consulting Laboratory, Department of Nursing, National Taipei College of Nursing, Taipei, Taiwan. ${ }^{6}$ Department of Pathology, National Taiwan University Hospital, Taipei, Taiwan. ${ }^{7}$ Department of Clinical Laboratory Sciences and Medical Biotechnology, College of Medicine, National Taiwan University, Taipei, Taiwan
\end{abstract}

\section{Conflict of interest}

The authors declare that they have no conflict of interest.

\section{Publisher's note}

Springer Nature remains neutral with regard to jurisdictional claims in published maps and institutional affiliations.

Supplementary Information accompanies this paper at (https://doi.org/ 10.1038/s41408-018-0074-7).

Received: 22 January 2018 Revised: 9 February 2018 Accepted: 15 February 2018

Published online: 04 April 2018

\section{References}

1. Tefferi, A. \& Vardiman, J. W. Myelodysplastic syndromes. N. Engl. J. Med. 361, 1872-1885 (2009).

2. Ades, L., Itzykson, R. \& Fenaux, P. Myelodysplastic syndromes. Lancet $\mathbf{3 8 3}$, 2239-2252 (2014).

3. Gangat, N., Patnaik, M. M. \& Tefferi, A. Myelodysplastic syndromes: contemporary review and how we treat. Am. J. Hematol. 91, 76-89 (2016).

4. Malcovati, L. et al. Time-dependent prognostic scoring system for predicting survival and leukemic evolution in myelodysplastic syndromes. J. Clin. Oncol. 25, 3503-3510 (2007).

5. Greenberg, P. et al. International scoring system for evaluating prognosis in myelodysplastic syndromes. Blood 89, 2079-2088 (1997).

6. Greenberg, P. L. et al. Revised international prognostic scoring system for myelodysplastic syndromes. Blood 120, 2454-2465 (2012).

7. Yoshida, K. et al. Frequent pathway mutations of splicing machinery in myelodysplasia. Nature 478, 64-69 (2011).

8. Bejar, R. et al. Clinical effect of point mutations in myelodysplastic syndromes. N. Engl. J. Med. 364, 2496-2506 (2011).

9. Langemeijer, S. M. et al. Acquired mutations in TET2 are common in myelodysplastic syndromes. Nat. Genet. 41, 838-842 (2009).

10. Raza, A. \& Galili, N. The genetic basis of phenotypic heterogeneity in myelodysplastic syndromes. Nat. Rev. Cancer 12, 849-859 (2012).

11. Papaemmanuil, E. et al. Clinical and biological implications of driver mutations in myelodysplastic syndromes. Blood 122, 3616-3627 (2013).

12. Haferlach, T. et al. Landscape of genetic lesions in 944 patients with myelodysplastic syndromes. Leukemia 28, 241-247 (2014).

13. Bejar, R. et al. Somatic mutations predict poor outcome in patients with myelodysplastic syndrome after hematopoietic stem-cell transplantation. J. Clin. Oncol. 32, 2691-2698 (2014).

14. Malcovati, L. et al. Driver somatic mutations identify distinct disease entities within myeloid neoplasms with myelodysplasia. Blood 124, 1513-1521 (2014).

15. Vardiman, J. W. et al. The2008 revision of the World Health Organization (WHO) classification of myeloid neoplasms and acute leukemia: rationale and important changes. Blood 114, 937-951 (2009).

16. Arber, D. A. et al. The 2016 revision to the World Health Organization classification of myeloid neoplasms and acute leukemia. Blood 127, 2391-2405 (2016).

17. Nazha, A. et al. Incorporation of molecular data into the Revised International Prognostic Scoring System in treated patients with myelodysplastic syndromes. Leukemia 30, 2214-2220 (2016). 
18. Chen, B. et al. Clinical and cytogenetic features of 508 Chinese patients with myelodysplastic syndrome and comparison with those in Western countries. Leukemia 19, 767-775 (2005).

19. Lee, J. H. et al. Application of different prognostic scoring systems and comparison of the $\mathrm{FAB}$ and $\mathrm{WHO}$ classifications in Korean patients with myelodysplastic syndrome. Leukemia 17, 305-313 (2003).

20. Yang, Y. T. et al. IPSS-R in 555 Taiwanese patients with primary MDS: integration of monosomal karyotype can better risk-stratify the patients. Am. J. Hematol. 89, E142-E149 (2014).

21. Bennett, J. M. et al. Proposals for the classification of the myelodysplastic syndromes. Br. J. Haematol. 51, 189-199 (1982).

22. Hou, H. A. et al. Clinical implications of the SETBP1 mutation in patients with primary myelodysplastic syndrome and its stability during disease progression. Am. J. Hematol. 89, 181-186 (2014).

23. Sanada, M. et al. Gain-of-function of mutated C-CBL tumour suppressor in myeloid neoplasms. Nature 460, 904-908 (2009).

24. Tang, J. L. et al. AML1/RUNX1 mutations in 470 adult patients with de novo acute myeloid leukemia: prognostic implication and interaction with other gene alterations. Blood 114, 5352-5361 (2009).

25. Hou, H. A. et al. GATA2 mutations in patients with acute myeloid leukemiapaired samples analyses show that the mutation is unstable during disease evolution. Ann. Hematol. 94, 211-221 (2015).

26. Hou, H. A. et al. Splicing factor mutations predict poor prognosis in patients with de novo acute myeloid leukemia. Oncotarget 7, 9084-9101 (2016).

27. Thol, F. et al. Frequency and prognostic impact of mutations in SRSF2, U2AF1, and ZRSR2 in patients with myelodysplastic syndromes. Blood 119, 3578-3584 (2012).

28. Lin, C. C. et al. IDH mutations are closely associated with mutations of DNMT3A, ASXL1 and SRSF2 in patients with myelodysplastic syndromes and are stable during disease evolution. Am. J. Hematol. 89, 137-144 (2014).

29. Hou, H. A. et al. DNMT3A mutations in acute myeloid leukemia: stability during disease evolution and clinical implications. Blood 119, 559-568 (2012).

30. Chou, W. C. et al. TET2 mutation is an unfavorable prognostic factor in acute myeloid leukemia patients with intermediate-risk cytogenetics. Blood 118 3803-3810 (2011)

31. Shiah, H. S. et al. Clinical and biological implications of partial tandem duplication of the MLL gene in acute myeloid leukemia without chromosomal abnormalities at 11q23. Leukemia 16, 196-202 (2002).
32. Chen, T. C. et al. Dynamics of ASXL1 mutation and other associated genetic alterations during disease progression in patients with primary myelodysplastic syndrome. Blood Cancer J. 4, e177 (2014)

33. Nikoloski, $\mathrm{G}$. et al. Somatic mutations of the histone methyltransferase gene EZH2 in myelodysplastic syndromes. Nat. Genet. 42, 665-667 (2010).

34. Tsai, C. H. et al. Prognostic impacts and dynamic changes of cohesin complex gene mutations in de novo acute myeloid leukemia. Blood Cancer J. 7, 663 (2017).

35. Hou, H. A. et al. Clinical implications of the SETBP1 mutation in patients with primary myelodysplastic syndrome and its stability during disease progression. Am. J. Hematol. 89, 181-186 (2013).

36. Hou, H. A. et al. TP53 mutations in de novo acute myeloid leukemia patients: longitudinal follow-ups show the mutation is stable during disease evolution. Blood Cancer J. 5, e331 (2015).

37. Tefferi, A. et al. Targeted next-generation sequencing in myelodysplastic syndromes and prognostic interaction between mutations and IPSS-R. Am. J. Hematol. 92, 1311-1317 (2017).

38. Bejar, R. et al. Validation of a prognostic model and the impact of mutations in patients with lower-risk myelodysplastic syndromes. J. Clin. Oncol. 30, 3376-3382 (2012).

39. Kennedy, J. A. \& Ebert, B. L. Clinical implications of genetic mutations in myelodysplastic syndrome. J. Clin. Oncol. 35, 968-974 (2017).

40. Walter, M. J. et al. Clonal architecture of secondary acute myeloid leukemia. $N$. Engl. J. Med. 366, 1090-1098 (2012).

41. Tefferi, A. et al. Mutations and karyotype in myelodysplastic syndromes: TP53 clusters with monosomal karyotype, RUNX1 with trisomy 21, and SF3B1 with inv(3)(q21q26.2) and del(11q). Blood Cancer J. 7, 658 (2017).

42. Walter, M. J. et al. Recurrent DNMT3A mutations in patients with myelodysplastic syndromes. Leukemia 25, 1153-1158 (2011).

43. Thol, F. et al. Prognostic significance of ASXL1 mutations in patients with myelodysplastic syndromes. J. Clin. Oncol. 29, 2499-2506 (2011).

44. Kulasekararaj, A. G. et al. TP53 mutations in myelodysplastic syndrome are strongly correlated with aberrations of chromosome 5, and correlate with adverse prognosis. Br. J. Haematol. 160, 660-672 (2013).

45. Cazzola, M., Della Porta, M. G. \& Malcovati, L. The genetic basis of myelodysplasia and its clinical relevance. Blood 122, 4021-4034 (2013).

46. Sperling, A. S., Gibson, C. J. \& Ebert, B. L. The genetics of myelodysplastic syndrome: from clonal haematopoiesis to secondary leukaemia. Nat. Rev. Cancer 17, 5-19 (2017). 